\title{
Influence of Bond Number on Behaviors of Liquid Drops Deposited onto Solid Substrates
}

\author{
Zhi-Qiang Zhu • Yang Wang • Qiu-Sheng Liu • \\ Jing-Chang Xie
}

Received: 18 April 2011 / Accepted: 10 December 2011 / Published online: 3 January 2012

(C) Springer Science+Business Media B.V. 2011

\begin{abstract}
The problem of spreading behaviors of pendant and sessile drops was studied experimentally and numerically under the action of gravity force and surface tension. Bond number was considered to be a main factor of the influence on shape behaviors of liquid drops. This study was performed in the framework of an experimental investigation of drop behaviors in microgravity onboard a Chinese satellite in future. The experiments were carried out in the Drop Tower of Beijing, which could supply about $3.6 \mathrm{~s}$ of microgravity (free-fall) time. The surface shape change of liquid drops was investigated and the contact angle variety in sessile and pendant drops were measured from normal gravity to microgravity. A sharp decrease and oscillatory variation of the contact angle for both sessile and pendant drops were found with the sudden decrease of Bond number. The succedent comparison between experimental and numerical results suggests that Bond number has a significant influence on the drop contact angle. Additionally, the drop shapes and the bulk flows inside sessile and pendant drops were analyzed numerically, and it was found that the bulk flows could affect the free-surface shape of liquid drops apparently. Comparison of the moving velocity of contact line between sessile and pendant drops indicated that the pendant drops had a faster response to Bond number.
\end{abstract}

Keywords Drop spreading - Bond number • Contact angle $\cdot$ Microgravity $\cdot$ Bulk flow

\footnotetext{
Z.-Q. Zhu · Y. Wang · Q.-S. Liu ( $\varangle)$ · J.-C. Xie

Key Laboratory of Microgravity (National Microgravity Laboratory), Institute of Mechanics, Chinese Academy of Sciences, Beijing 100190, China

e-mail: liu@imech.ac.cn
}

\section{Introduction}

In the past decades, with the development of the industry, liquid drop has attracted growing academic interests due to its varieties of applications in fundamental and industrial fields. Generally, drops have two existing status: suspending and attaching to solid substrates, the latter was paid more attention due to its ubiquity in universe. Owing to independent directions of practical requests, liquid drop has been studied in different aspects. These studies involve drop evaporating process (Grandas et al. 2005; Shahidzadeh-Bonn et al. 2006), influences to contact angles (Bourges-Monnier and Shanahan 1995; Crafton and Black 2003; Panwar et al. 2003), moving contact lines (Dussan 1979; Vladimir 2005), thermocapillary convection inside drops (Ruiz and Black 2002; Savino et al. 2002), migration of drops (Greenspan 1978; Smith 1995) and spreading of liquid drops on the solid substrates (Cox 1986). In the present paper, we are focusing on the spreading of liquid drops on a horizontal plate.

Spreading of liquid drops on a solid surface has broad applications in industrial and biological fields, such as coating, painting and cell sorting. Recently, spreading of liquid drops was found to play an obvious influence on the liquid lubrication of moving mechanical assemblies (MMA). Motivated by above mentioned applications, a number of experimental and numerical works have been carried out to study the spreading behaviors of drops under different conditions. Consideration of drop spreading on a solid substrate will be useful for deeper understanding of the wetting phenomena. Generally, the shapes of liquid drops attached to solid substrates could be influenced by many factors. In present work, only gravity effect and surface tension 
are taken into account to study the spreading behaviors of liquid drops with the sudden change of gravity effect from $1 \mathrm{~g}$ to microgravity.

Actually, the most important parameter governing drop spreading behaviors and related heat transfer capability is the drop contact angle, which is also being widely used to described drop-related phenomena. Apparently, the contact angle of liquid drop was defined to reflect the dynamic equilibrium between the three phases: liquid phase of the drop, solid phase of the substrate, and vapor phase of the ambient air. Therefore, the vapor-liquid-solid triple line, i. e. the contact line of drop, is usually considered to be strongly related to the contact angle. Ponter and Boyes (1972) firstly studied the contact angle of sessile liquid drop. They found that only small drops (smaller than $5 \mathrm{~mm}$ ) changed their contact angles under variable drop diameter. Subsequently, a number of experimental and numerical studies have been performed since Hoffman's experimental finding (Hoffman 1975) which suggested a universal relation between the contact line motion velocity and drop contact angle. However, succedent studies of Hocking (1983) and Cox (1986) both demonstrated that the Hoffman's law was only valid for small Bond numbers and it could not predict the drop spreading behaviors for large Bond numbers. Follow-up researches (Fermigier and Jenffer 1991; Hayes and Ralston 1993) all yielded similar conclusions supporting Cox's finding.

Owing to the fact that bulk flow could influence the temperature distribution and free surface of liquid drops, bulk flow inside the liquid drop is thought to be another main influence to affect the drop contact angle. Bigioni et al. (2006) found that the convection inside the drop made the particles easily to accumulate in the vicinity of the triple line. Hu and Larson (2005), Girard et al. (2008) carried out numerical simulations to study marangoni flow in sessile evaporating drops. And they found different temperature distributions inside the drops comparing to the results that neglect inner flow. Based on previous literatures, many studies concerning the contact line and contact angle were mainly developed under the condition of drop evaporating. In present paper, we have neglected the evaporation effect, and only gravity effect and surface tension were involved to investigate the drop spreading behaviors under a sudden change of Bond number.

No doubts that many research works have been carried out to provide much understanding of drop spreading behaviors under different conditions. Nevertheless, the mechanism of drop spreading process is complex and still absent of comprehensive understanding even if it has been widely used in industry. In above mentioned literatures, all the experimental and numerical research are carried out under normal gravity. Actually, a different drop spreading behavior in microgravity has been found (Brutin et al. 2009). A better understanding and clear knowledge of the physical mechanism of drop spreading in microgravity could widen the applications of liquids drops with the development of space industry.

In presented paper, a combined experimental and numerical investigation is carried out to study the spreading behaviors of sessile and pendant drops influenced by the gravity force, which is represented making use of Bond number. In what follows, we will first specify the description of the experimental apparatus and the measurement techniques in "Experimental Setup and Measurement Techniques". The numerical model is presented detailedly in "Simulation Model". In "Results and Discussion", we will present and discuss the experimental and numerical results, including the shape and contact angles behaviors depending on varying Bond number, influence of bulk flow in liquid drop on the free surface and contact angle in different conditions. In addition, comparison will be performed between the experimental and numerical results to testify the validity of numerical model. Moreover, a preliminary comparison of moving line velocity between pendant and sessile drops will also be numerically analyzed to investigate the response of Bond number for different drops. Finally, comments and conclusions are stated in "Conclusion".

\section{Experimental Setup and Measurement Techniques}

All the experiments were performed in the Drop Tower of Beijing, which could supply about $3.6 \mathrm{~s}$ of microgravity (free-fall) time. The experimental apparatus used in this study has already been previously described in Zhu et al. (2010). Briefly, two substrates (each $10 \mathrm{~mm}$ in diameter) made of aluminum were put into a test cell, of which the sidewalls were made of plexiglass for optics observations and outer air insulation. In our experiments, the two substrates were usually covered by two kinds of top surfaces, aluminum and polytetrafluoroethylene (PTFE), respectively, to analyze the wetting behaviors of liquid drops for different attached solid surface. During the experiments, two calibrated unsheathed fine-gauge thermocouples (type $\mathrm{T}), 25.4 \mu \mathrm{m}$ in diameter, with an accuracy of $\pm 0.02^{\circ} \mathrm{C}$ and a rapid response time of $0.05 \mathrm{~s}$, were respectively inserted into a hole $5 \mathrm{~mm}$ below each top surface of the substrate to monitor the temperature, which was fed back to the PID controller and heating cartridge to control the surface temperature of the substrate with an accuracy of $0.05^{\circ} \mathrm{C}$. 
As we know, drop contact angle is directly related to properties of the substrate, such as roughness, surface cleanness. In present experiments, the two substrates were covered by two kinds of top surfaces, aluminum and PTFE, respectively, which were thin smooth sheets with a thickness of $200 \mu \mathrm{m}$. Additionally, motivated by the request of performing all the experiments with same clean surface condition, prior to each experiment, the surfaces were sequentially cleaned with acetone, ethanol and de-ionized water. The surfaces were then dried with lens cleaning paper and finally flushed with compressed air to remove remaining wastepaper or dust.

De-ionized water was used in these experiments due to its ubiquity in nature and bad wettability for PTFE and aluminum, which means relatively bigger drop with bigger contact angle could be created for easier observation and analysis. Reversely, water is sensitive to contamination, which could influence the contact angle of drop apparently. To perform all the experiments with same clean surface condition, prior to each experiment, the solid surfaces were carefully cleaned and dried to remove impurity or dust.

After reaching top of the drop tower, firstly, deionized water was transferred directly into two syringes mounted in a syringe pump. Together with a step motor and a PLC controller, drops were then injected and created through a $700 \mu \mathrm{m}$ hole inside the substrate at regulated injection rate and volume. After liquid drops and surface temperature getting stable, the experimental apparatus fell down, i.e. the drops entering microgravity. And CCD camera began to record the shape change of drops for computer analysis. Whereafter, through the obtained images, a software program (Yu et al. 2009) was introduced to measure and determine contact angle of the drops with an accuracy of $\pm 3^{\circ}$.

\section{Simulation Model}

Simulation of the above experiments was carried out by means of VOF methodology. The simulation model of a liquid drop on a horizontal plate is shown in Fig. 1.

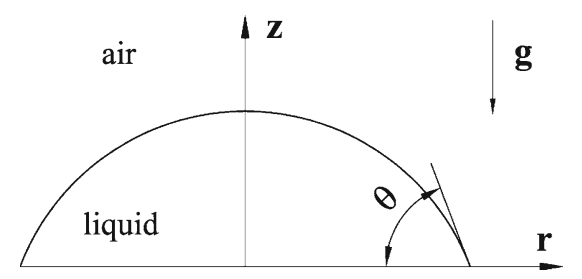

Fig. 1 Simulation model of a drop spreading on a horizontal surface
Cylindrical coordinates $(r, z)$ are chose with the axis $\mathrm{Or}$ being horizontal direction and the axis $O z$ parallel to gravity $g$ direction. Note that the gravity $g$ direction is different for sessile and pendant drop. Additionally, the liquid drop is surrounded by air. The numerical study has been performed with the following assumptions:

1. The liquid drop on a horizontal plate is considered as two-dimensional and axisymmetric. And we can only simulate half of the drop to reduce the calculation time.

2. The evaporation effect is negligible. And the drop volume is constant.

3. The liquid density is independent on the temperature.

4. The temperature has no influence on the viscosity of liquid.

5. The liquid is incompressible and the outer air is compressible.

The momentum, continuity and energy equations are expressed in the form:

$$
\begin{aligned}
& \frac{\partial \mathbf{u}_{r}}{\partial t}+\mathbf{u}_{r} \frac{\partial \mathbf{u}_{r}}{\partial t}+\mathbf{v}_{z} \frac{\partial \mathbf{u}_{r}}{\partial z} \\
& =-\frac{1}{\rho} \frac{\partial p}{\partial r}+v\left(\frac{1}{r} \frac{\partial \mathbf{u}_{r}}{\partial r}+\frac{\partial^{2} \mathbf{u}_{r}}{\partial r^{2}}+\frac{\partial^{2} \mathbf{u}_{r}}{\partial z^{2}}-\frac{\mathbf{u}_{r}}{r^{2}}\right) \\
& \frac{\partial \mathbf{v}_{z}}{\partial t}+\mathbf{u}_{r} \frac{\partial \mathbf{v}_{z}}{\partial t}+\mathbf{v}_{z} \frac{\partial \mathbf{v}_{z}}{\partial z} \\
& =-\frac{1}{\rho} \frac{\partial p}{\partial z}+v\left(\frac{1}{r} \frac{\partial \mathbf{v}_{z}}{\partial r}+\frac{\partial^{2} \mathbf{v}_{z}}{\partial r^{2}}+\frac{\partial^{2} \mathbf{v}_{z}}{\partial z^{2}}\right) \\
& \frac{\partial \mathbf{u}_{r}}{\partial r}+\frac{\mathbf{u}_{r}}{r}+\frac{\partial \mathbf{v}_{z}}{\partial z}=0 \\
& \frac{\partial F}{\partial t}+\frac{\partial\left(\mathbf{u}_{r} F\right)}{\partial r}+\frac{\partial\left(\mathbf{v}_{z} F\right)}{\partial z}=0
\end{aligned}
$$

where $\mathbf{u}_{r}\left(\mathbf{v}_{z}\right), p$ and $F$ are velocity, pressure and VOF function. $\rho, v$ are the density and kinematic viscosity.

The Bond number is defined as

$$
B o=\frac{\rho g h^{2}}{\sigma}
$$

where $g$ is the gravity acceleration, $h$ is the height of the liquid drop, and $\sigma$ is the surface tension of liquid.

The volume of fluid (VOF, Hirt and Nichols 1981) method is an efficient methodology to simulate the system consisting of two separate phases. In this method, a scalar function $\mathrm{F}$ is defined as the fraction of a cell volume occupied by fluid. $\mathrm{F}$ is assumed to be unity when the cell is fully occupied by the fluid and zero for an empty cell. Cells with values of $0<\mathrm{F}<1$ contain a 


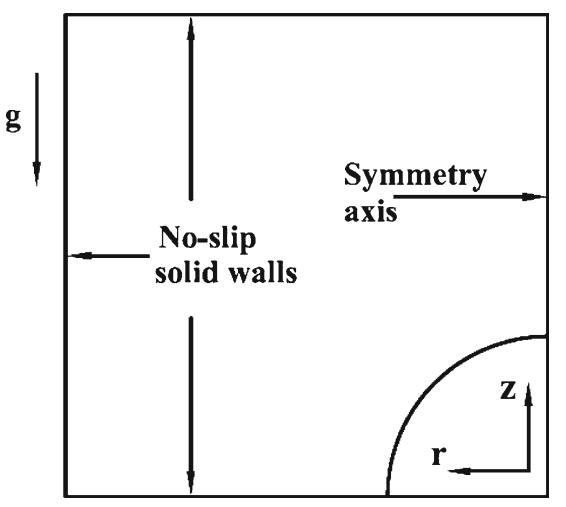

Fig. 2 Simulated domain and boundary conditions for the liquid drop

free surface. In present work, only half of liquid drop was simulated due to assumption of axisymmetric. The rectangle computational domain was chosen to be three times of drop scale after testing. Quadrangular grids $0.05 \mathrm{~mm}$ between each were used in the computational domain. The boundary conditions are shown is Fig. 2, and the temperatures of solid walls were set equal to the ambient temperature.

\section{Results and Discussion}

The experiments were carried out in the framework of obtaining reference data on the liquid drop behaviors in microgravity for future space experiment in Chinese satellite. Therefore, the liquid and drop size used in present study were chosen according to the parameters of the future space experiment.

The spreading behaviors of liquid drops attached to solid substrates involve many influences, such as wettability, surface tension, Bond number, bulk flow and moving contact line. Making use of the above mentioned experimental apparatus and measuring techniques, drop shape and contact angle were measured to reflect the spreading behaviors of both sessile and pendant drops from normal gravity to microgravity.

For comparison, simulation must be performed under the same conditions with experiments. In present simulation, volume of the liquid drop was firstly determined based on the actual volume of corresponding experiment. The actual drop contact angle in normal gravity, i.e. drop contact angle at the beginning of the experiment, was also measured to be the initial drop contact angle of the simulation. Subsequently, based on the stresses balance at the free surface of liquid drops, the drop contact angle change during the process of drop entering microgravity could be simulated.

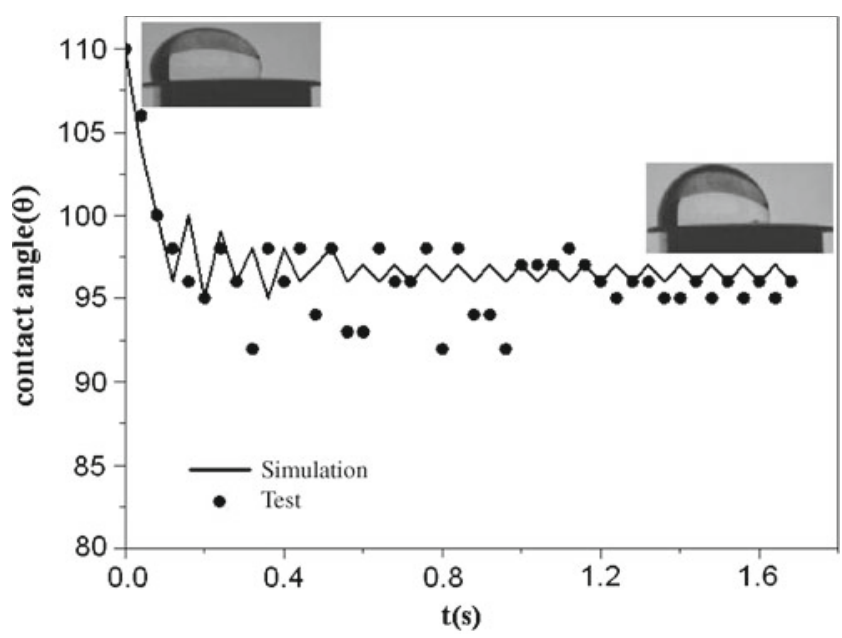

Fig. 3 Contact angle change of sessile water drop on PTFE from normal gravity to microgravity

Contact Angle Behaviors in Microgravity

As we know, drop contact angle is very sensitive to gravity level. Figs. 3 and 4 show respectively contact angle changes of sessile water drop on PTFE and pendant water drop on aluminum during one experiment, i. e. the gravity level changes from normal to microgravity. The experimental results are denoted as black circle dots. Additionally, simulated results are also drawn in these two figures.

For sessile drop experiment (seen in Fig. 3), a water drop, $7.4 \mathrm{~mm}$ in diameter and $0.103 \mathrm{~mL}$ in volume, was injected and created at normal gravity with an injecting rate of $17 \mu L / s$. The shape variations were recorded

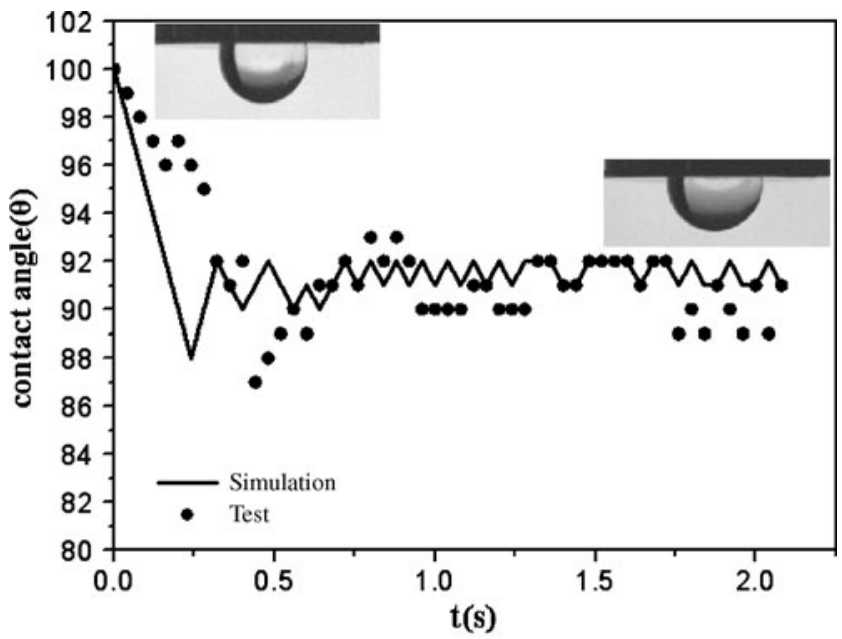

Fig. 4 Contact angle change of pendant water drop on aluminum from normal gravity to microgravity 
as soon as entering microgravity, that is, Bond number suddenly decreased from 7 to $7 \mathrm{e}-5$. It could be seen that the drop contact angle has a sudden decrease from $110^{\circ}$ to $95^{\circ}$, following the sudden decrease of Bond number. Referring to experimental pictures in Fig. 2, a rapid increase of height of the drop was also observed, which was accordant to former numerical results (Reznik and Yarin 2002). Following the sudden change, shape of the drop began to oscillate, with contact angle varying from $98^{\circ}$ to $92^{\circ}$. After $1.2 \mathrm{~s}$, surface tension stabilized the drop with a final contact angle of $96^{\circ}$. For pendant water drop on aluminum (shown in Fig. 4), after creating a water drop with the diameter, volume and injecting rate of $3.0 \mathrm{~mm}, 0.02 \mathrm{~mL}$ and $10 \mu \mathrm{L} / \mathrm{s}$, respectively, the same sudden change of drop contact angle could be seen from $100^{\circ}$ to $86^{\circ}$ as soon as entering microgravity. After a $1.0 \mathrm{~s}$ oscillation, the drop contact angle stabilized at $90^{\circ}$. Based on above description, it is obvious that no matter the water drop on PTFE or on aluminum, the contact angles in normal gravity are always bigger than that in microgravity. Taking sessile water drop for example, the gravity effect and surface tension influence the drop shape together. And pressing of the gravity effect and pinning of the drop contact line ultimately press the liquid close to the contact line downwards and outwards. After entering microgravity, disappearing of the gravity effect releases the liquid close to the contact line to rebound upwards, which decreases the drop contact angle. Through Comparison, it could be found that numerical results were approximately consistent with experimental results, except drop contact angle stable times in simulation were shorter than that in experiments, with $0.8 \mathrm{~s}$ to $1.2 \mathrm{~s}$ for sessile drops, and $0.7 \mathrm{~s}$ to $1.0 \mathrm{~s}$ for pendant drops. Anyhow, the consistence with experimental results also suggests that our simulation is an efficient way to predict the spreading behaviors of liquid drops attached to solid substrates under the influence of Bond number.
Fig. 5 Sessile water drops on PTFE: Bulk flows inside drops in microgravity. a 0.001 s, b $0.01 \mathrm{~s}$, c $0.02 \mathrm{~s}, \mathbf{d} 0.036 \mathrm{~s}$
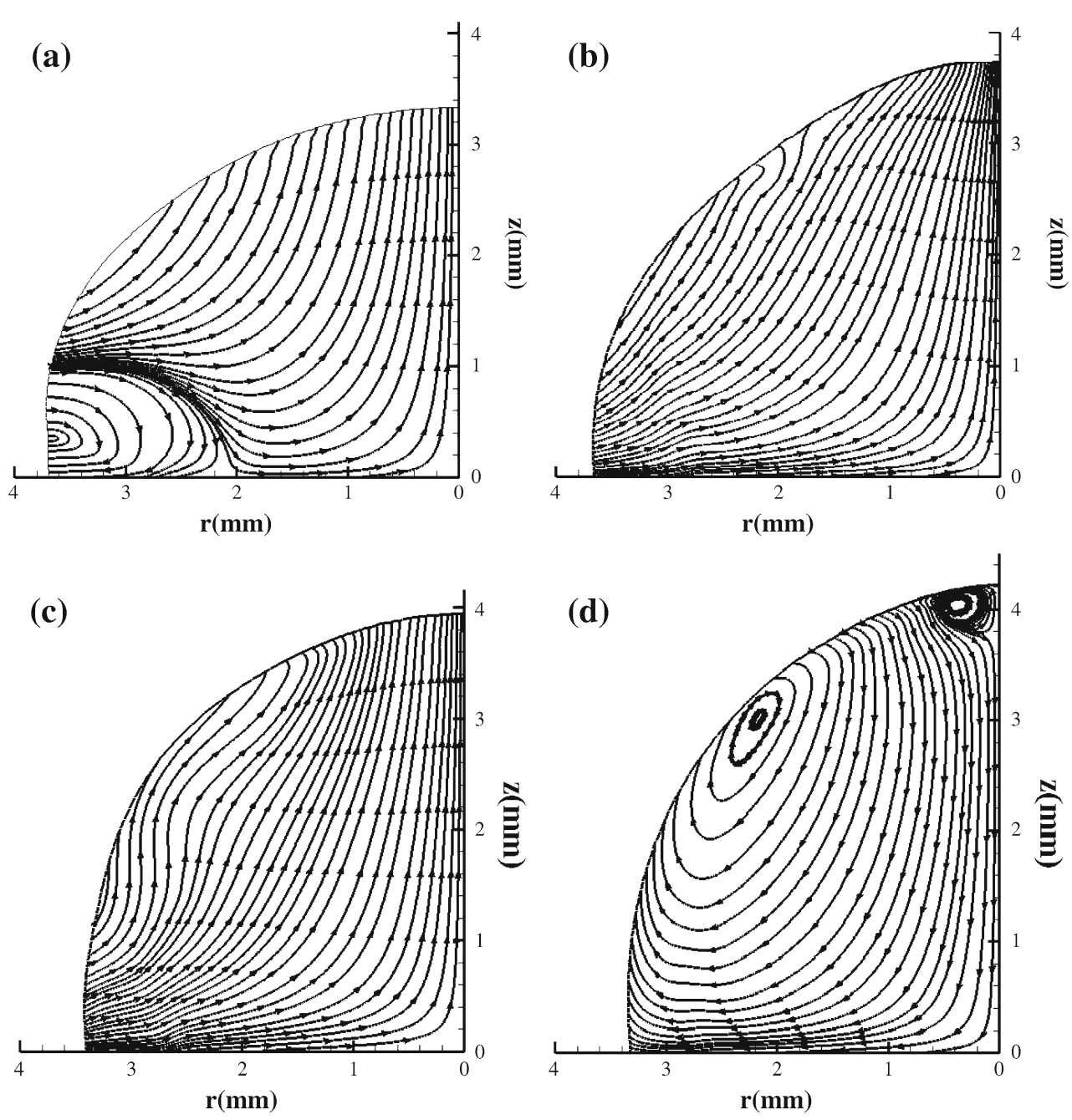

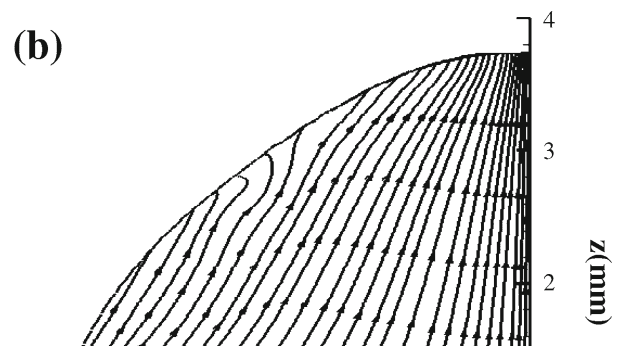

츨 
Bulk Flows Inside Drops in Microgravity

According to previous literatures, the shape of liquid drop attached to solid substrate is mainly determined by the stresses balance at the free surface of drop. And the balance could be influenced by gravity effect, surface tension and bulk flow inside the liquid drop. Actually, gravity effect together with surface tension, i.e. Bond number, could also induce the bulk flow, which could eventually influence the contact line and free surface shape of the liquid drop. Figs. 5 and 6 show the bulk flows inside sessile water drop on PTFE and pendant water drop on aluminum as soon as entering microgravity, respectively.

As shown in Fig. 5, with volume of $0.103 \mathrm{~mL}$ and initial contact angle of $110^{\circ}$, after Bond number sudden decreased from 7 to $7 \mathrm{e}-5$, it could be seen that following the sudden decrease of Bond number (Fig. 5a), gravity vanished, and decrease of contact angle made the triple line to spread, which means the liquid close to triple line flow outwards. Simultaneously, inertia force made the liquid to flow upwards (Fig. 5b). It is obvious that inertia force and contact angle play opposite influence on the bulk flow direction inside the drop. Actually, inertia force played a more important role than contact angle in this case. As a result, the flow inside the drop had an overall trend of flowing upwards, which induced increase of the drop height and decrease of the drop radius, shown in Fig. 5c. After the drop reaching the highest point, surface tension would pull the drop back, and there would be a flow downwards, shown in Fig. 5d. Similarly, inertia force would influence liquid to flow upwards again after the drop height reaching the lowest point. And there will be an oscillation of the drop shape. Eventually, the drop would stay stable after the balance of inertia effect and surface tension.
Fig. 6 Pendant water drops on aluminum: Bulk flows inside drops in microgravity. a $0.001 \mathrm{~s}, \mathbf{b} 0.01 \mathrm{~s}, \mathbf{c} 0.02 \mathrm{~s}$, d $0.04 \mathrm{~s}$

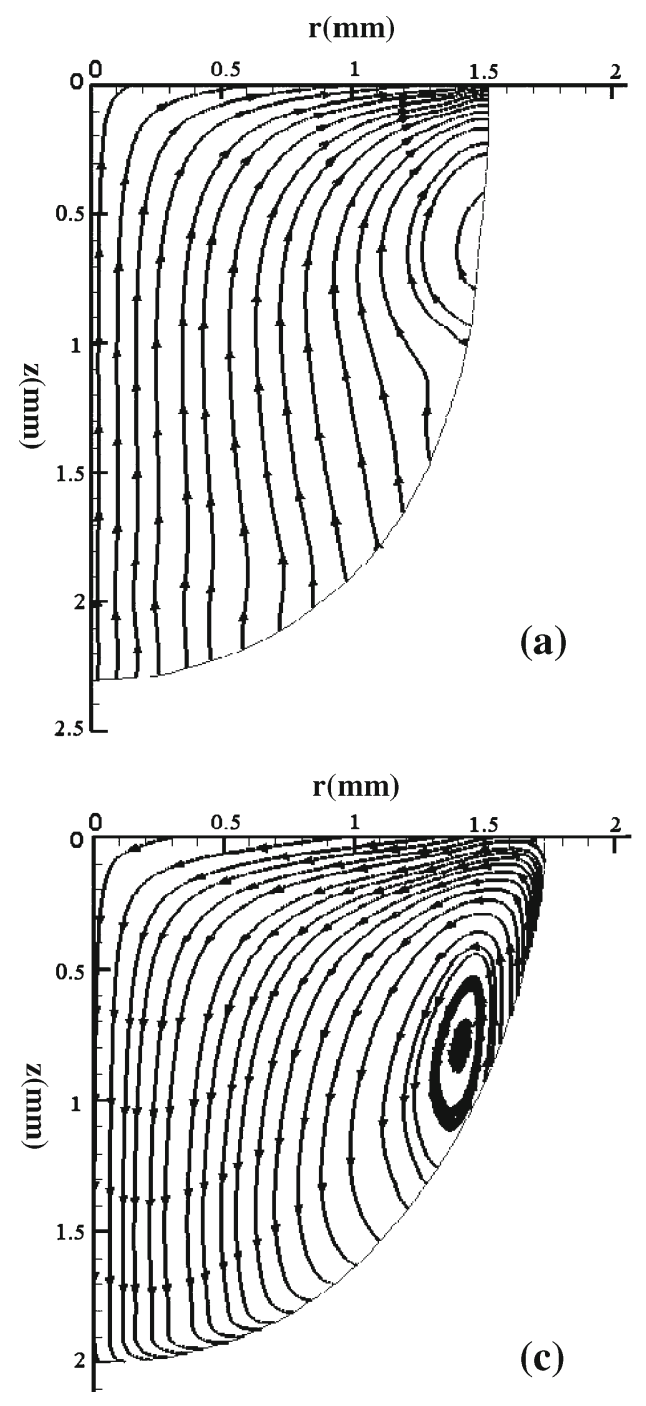

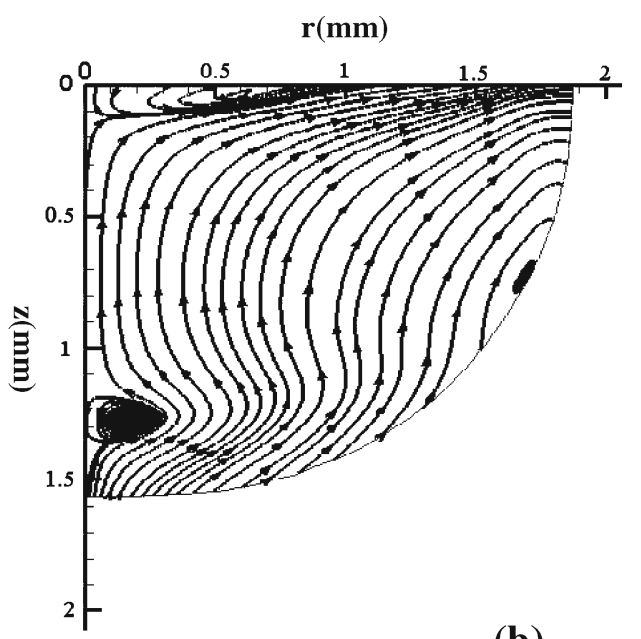

(b)

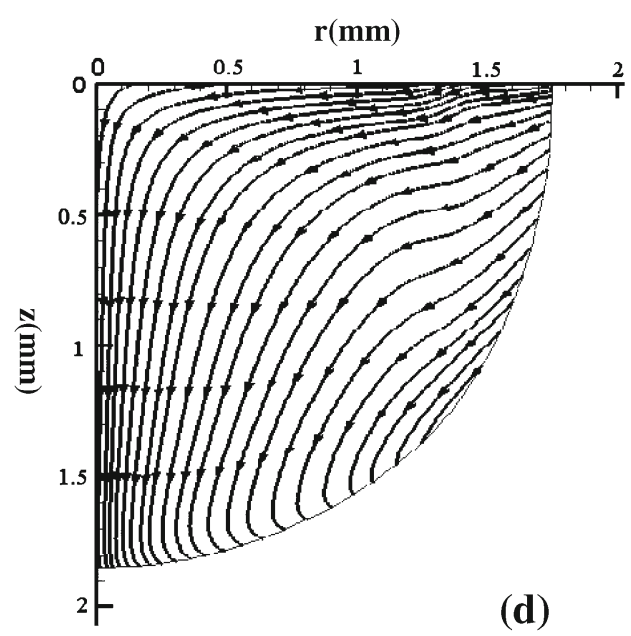


Similar trend could be found for pendant drop with volume of $0.02 \mathrm{~mL}$ and initial contact angle of $100^{\circ}$ in Fig. 6. Differently with the sessile drop, following the sudden decrease of Bond number, inertia force and decreasing of contact angle both made the drop to spread outwards (Fig. 6a, b), which induced decrease of the drop height and increase of the drop radius. After the drop reaching the highest point, the contact line would turn back, and there would be a flow inwards, shown in Fig. 6c. Similarly, after an oscillation of the drop shape, the drop would stay stable eventually (Fig. 6d).

\section{Comparison Between Sessile and Pendant Drops}

According to above experimental and simulated results, it is obvious that no matter sessile or pendant drop, coupling of gravity effect and surface tension, $i$. e. Bond number, has apparent influence on the free surface and contact line of liquid drops. Based on the contact angle behaviors in microgravity (seen in Figs. 3, 4), experiments and simulations all support the fact that stabilized times of sessile drop are always longer than those of pendant drop. In order to compare the spreading behaviors between sessile and pendant drops, the moving velocity of contact line was simulated and presented in Fig. 7.

It is seen that the contact line begins to move as soon as entering microgravity, and the moving velocity of contact line subsequently oscillate due to coupling of gravity effect and surface tension. For pendant drop, the oscillation time of $0.3 \mathrm{~s}$ is shorter than that of sessile drop. It is supposed that the opposite influence of gravity effect and surface tension in sessile drop lengthens

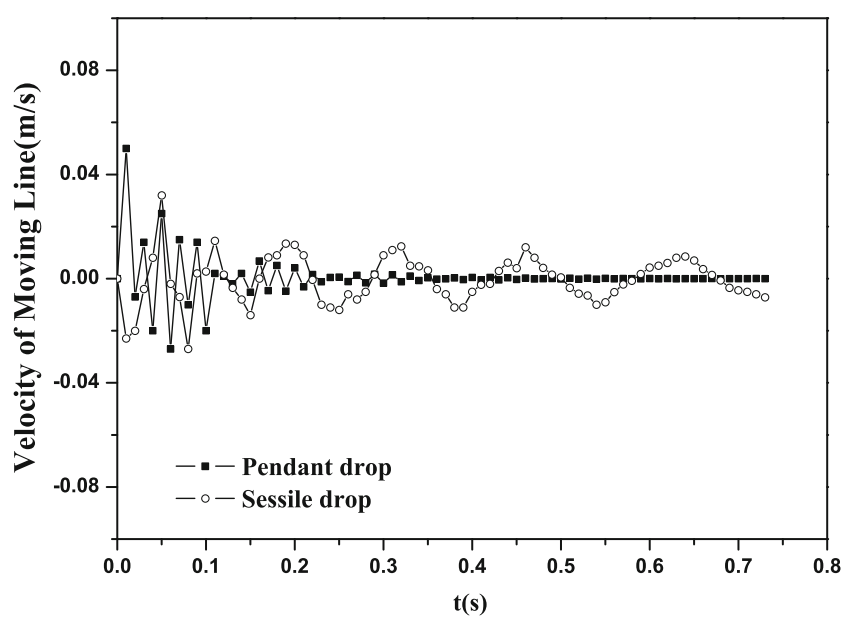

Fig. 7 Simulation for moving velocity of contact line the stabilized process. Therefore, we can infer that pendant drop has a faster response to Bond number than sessile drop with the liquid drop entering microgravity from normal gravity. Note that owing to liquid drop's nearly pinning on solid substrate, the contact line stabilizes faster than the contact angle $(1.0 \mathrm{~s}$ for pendant drop), which is synchronously related to the free surface moving of the drop.

\section{Conclusion}

The spreading behaviors of sessile and pendant drops deposited onto horizontal solid substrates were studied experimentally and numerically under the action of gravity force and surface tension, i.e. under the influence of Bond number. The contact angle, bulk flow and moving contact line were taken into account to study the shape behaviors of liquid drops.

A sharp decrease of the contact angle was found as soon as the drop entering microgravity from normal gravity, accompanying with the sudden decrease of Bond number. And a succedent oscillation of contact angle was found due to coupling of inertia and surface tension. Comparing with experimental results, the approximate consistence also testified the simulation's validity. Through the simulation of bulk flows inside liquid drops, it was found that the contact line firstly spread outwards due to the decrease of contact angle. Subsequently, inertia effect and surface tension would coupled to influence the bulk flow, which induced an oscillation of drop shape. Eventually, the drop would stay stable after the balance of inertia force and surface tension. Comparison on moving velocity of contact line was performed between sessile and pendant drops, it was inferred that pendant drop had a faster response to Bond number than sessile drop, with the oscillation time of pendant drop shorter than that of sessile drop. And it is supposed that the opposite influence of gravity effect and surface tension in sessile drop lengthens the stabilized process. The more details of experimental and numerical investigation on the spreading behaviors of liquid drops will be performed to focus on coupling of other influences besides Bond number, in a framework of future space experiments.

Acknowledgements This research was financially supported by the National Natural Science Foundation of China (Grant Nos. 11072249 and 50890182) and the Knowledge Innovation Project of Chinese Academy of Sciences (CXJJ-10-M55), the Knowledge Innovation Project of Institute of Mechanics/CAS. 


\section{References}

Bigioni, T.P., Lin, X.M., Nguyen, T.T., Corwin, E.I., Witten, T.A., Jaeger, H.M.: Kinetically driven self assembly of highly ordered nanoparticle monolayers. Nat. Mater. 5, 265-270 (2006)

Bourges-Monnier, C., Shanahan, M.E.R.: Influence of evaporation on contact angle. Langmuir 11, 2820-2829 (1995)

Brutin, D., Zhu, Z.Q., Rahli, O., Xie, J.C., Liu, Q.S., Tadrist, L.: Sessile drop in microgravity: creation, contact angle and interface. Microgravity Sci. Technol. 21(Suppl 1), S67-S76 (2009)

Cox, R.G.: The dynamics of the spreading of liquids on a solid surface. Part 1. Viscous flow. J. Fluid Mech. 168, 169-194 (1986)

Crafton, E.F., Black, W.Z.: Heat transfer and evaporation rates of small liquid droplets on heated horizontal surfaces. Int. J. Heat Mass Transfer 47, 1187-1200 (2003)

Dussan, E.B.: On the spreading of liquids on solid surfaces: static and dynamic contact lines. Ann. Rev. Fluid Mech. 11, 371-400 (1979)

Fermigier, M., Jenffer, P.: An experimental investigation on the dynamic contact angle in liquid-liquid systems. J. Colloid Interface Sci. 146, 226-241 (1991)

Girard, F., Antoni, M., Sefiane, K.: On the effect of marangoni flow on evaporation rates of heated water drops. Langmuir 24, 9207-9210 (2008)

Grandas, L., Reynard, C., Santini, R., Tadrist, L.: Experimental study of the evaporation of a sessile drop on a heat wall. Int. J. Therm. Sci. 44(2), 137-146 (2005)

Greenspan, H.P.: On the motion of a small viscous droplet that wets a surface. J. Fluid Mech. 84(1), 169-194 (1978)

Hayes, R.A., Ralston, J: Forced liquid movement on law energy surfaces. J. Colloid Interface Sci. 159, 429-438 (1993)

Hirt, C.W., Nichols, B.D.: Volume of fluid (VOF) method for the dynamics of free boundries. J. Comput. Phys. 39(1), 201-225 (1981)
Hocking, L.M.: The spreading of a thin drop by gravity and capillarity. Q. J. Mech. Appl. Math. 36, 55-69 (1983)

Hoffman, R.: A study of the advancing interface. I. Interface shape in liquid-gas systems. J. Colloid Interface Sci. 50, 228 241 (1975)

Hu, H., Larson, R.G.: Analysis of the effect of marangoni stresses on the microflow in an evaporating sessile drop. Langmuir 21, 3972-3980 (2005)

Panwar, A.K., Barthwal, S.K., Ray, S.: Effect of evaporation on the contact angle of a sessile drop on solid substrates. J. Adhes. Sci. Technol. 17(10), 1321-1329 (2003)

Ponter, A.B., Boyes, A.P.: The relation between contact angle and drop size for water at its boiling point for a pressure range 50 C760 Torr. Can. J. Chem. 50, 2419 (1972)

Reznik, S.N., Yarin, A.L.: Spreading of an axisymmetric viscous drop due to gravity and capillarity on a dry horizontal wall. Int. J. Multiph. Flow 28, 1437-1457 (2002)

Ruiz, O.E., Black, W.Z.: Evaporation of water droplets placed on a heated horizontal surface. J. Heat Transfer 124, 854-863 (2002)

Savino, R., Paterna, D., Favaloro, N.: Buoyancy and marangoni effects in an evaporating drop. J. Thermophys. Heat Transf. 16(4), 562-574 (2002)

Shahidzadeh-Bonn, N., Rafai, S., Azouni, A., Bonn, D.: Evaporating droplets. J. Fluid Mech. 549, 307-313 (2006)

Smith, M.K.: Thermocapillary migration of a two-dimensional liquid droplet on a solid surface. J. Fluid Mech. 294, 209-230 (1995)

Vladimir, S.A.: Spreading of thin volatile liquid droplets on uniformly heated surfaces. J. Fluid Mech. 528, 279-296 (2005)

Yu, Q., Cai, S.J., Zhu, Z.Q., Liu, Q.S., Zhou, B.H.: Droplet image feedback control system in evaporation experiment. Microgravity Sci. Technol. 22, 139-144 (2009)

Zhu, Z.Q., Brutin, D., Liu, Q.S., Wang, Y., Mourembles, A., Xie, J.C., Tadrist, L.: Experimental investigation of pendant and sessile drops in microgravity. Microgravity Sci. Technol. 22, 339-345 (2010) 